\title{
Electronic and Transport Properties of Sr-site Substituted: $\mathrm{Ca}_{\mathrm{x}} \mathrm{Sr}_{(1-\mathrm{x})} \mathrm{VO}_{3}$
}

\author{
R. K. Rai, R. B. Ray, G. C. Kaphle, O. P. Niraula
}

Journal of Nepal Physical Society

Volume 7, Issue 1, April 2021

(Special Issue: ANPA Conference, 2020)

ISSN: 2392-473X (Print), 2738-9537(Online)

\section{Editors:}

Dr. Santosh KC

San Jose State University, USA (Editor in Chief)

Dr. Pashupati Dhakal

Thomas Jefferson National Accelerator Facility, USA

Dr. Yadav Pandit

Baptist Health Science University, USA

\section{Managing Editor:}

Dr. Binod Adhikari

St. Xavier's College, Kathmandu, Nepal

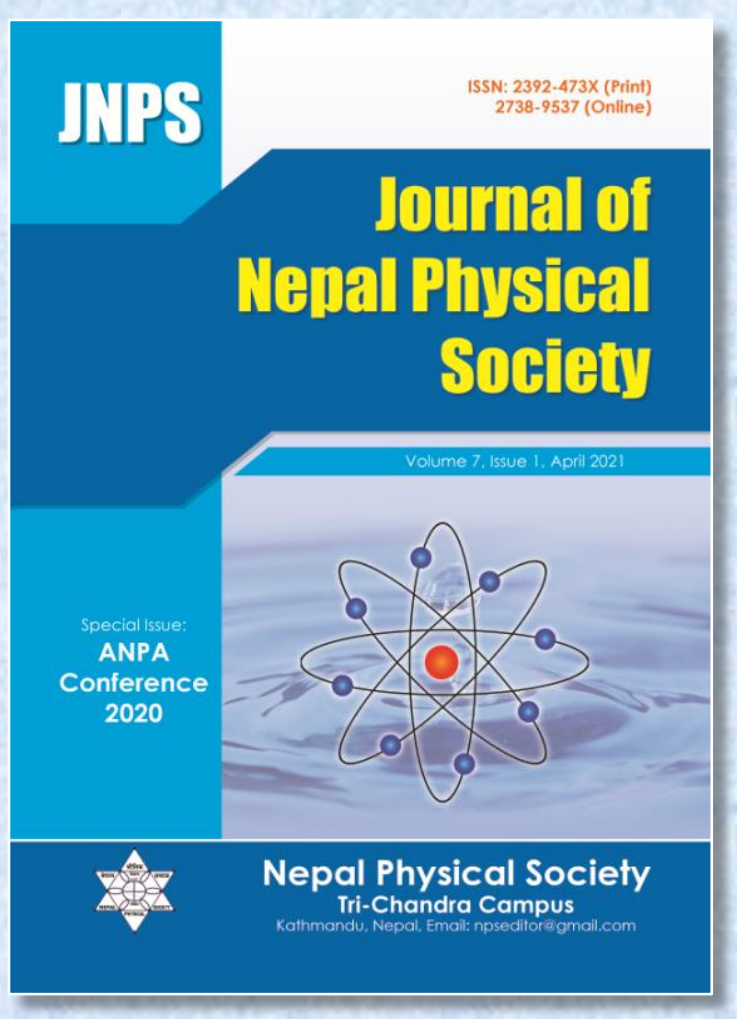

JNPS, 7 (1), 6-17 (2021)

DOI: http://doi.org/10.3126/jnphyssoc.v7i1.36968

\section{Published by:}

Nepal Physical Society

P.O. Box: 2934

Tri-Chandra Campus

Kathmandu, Nepal

Email:npseditor@gmail.com 


\title{
Electronic and Transport Properties of Sr-site Substituted: $\mathrm{Ca}_{\mathbf{x}} \mathrm{Sr}_{(1-\mathrm{x})} \mathrm{VO}_{3}$
}

\author{
R. K. Rai ${ }^{1,2}$, R. B. Ray ${ }^{1,3}$, G. C. Kaphle ${ }^{1, *}$, O. P. Niraula ${ }^{1}$ \\ ${ }^{1}$ Central Department of Physics, Tribhuvan University, Kirtipur, Kathmandu, Nepal \\ ${ }^{2}$ Patan Multiple Campus, Tribhuvan University, Lalitpur, Nepal \\ ${ }^{3}$ Amrit Campus, Tribhuvan University, Kathmandu, Nepal \\ *Corresponding Email: gck223@gmail.com
}

Received: 9 August, 2020; Revised: 10 January, 2021; Accepted: 5 February, 2021

\begin{abstract}
The Mott-insulator phase transition behaviour of the superstructure of strongly correlated system, $\mathrm{Ca}_{\mathrm{x}} \mathrm{Sr}_{(1-\mathrm{x})} \mathrm{VO}_{3}(\mathrm{x}=0,0.33,0.67,1)$ have studied using the conventional density functional theory and the dynamical mean field theory. The Mott-Hubbard metal-insulator phase transition of superstructures, $\mathrm{Ca}_{0.33} \mathrm{Sr}_{0.67} \mathrm{VO}_{3}$ and $\mathrm{Ca}_{0.67} \mathrm{Sr}_{0.33} \mathrm{VO}_{3}$ formed by the $\mathrm{CaVO}_{3}$ and $\mathrm{SrVO}_{3}$ correlated metals, are obtained at $\mathrm{U}=4.5 \mathrm{eV}$ with $\beta=6(\mathrm{eV})^{-1}$ and $\mathrm{U}=4.5 \mathrm{eV}$ with $\beta=$ $7(\mathrm{eV})^{-1}$ respectively. The values of $U$ and $\beta$ calculated through the Maximum Entropy model using the Green's function data, are consistent with the experimental results. The value of Seebeck coefficient (S) of superstructure $\mathrm{Ca}_{0.33} \mathrm{Sr}_{0.67} \mathrm{VO}_{3}$ and $\mathrm{Ca}_{0.67} \mathrm{Sr}_{0.33} \mathrm{VO}_{3}$ are found to be $+0.0011[\mathrm{~V} / \mathrm{K}]$ and $-0.0011[\mathrm{~V} / \mathrm{K}]$ within the chemical potential $\mu=-1.266 \mathrm{eV}$ to $\mu=$ $-0.938 \mathrm{eV}$. The figures of merit (ZT) are found to be 0.97 at room temperature for these systems. The variation of electrical and thermal conductivities has also been discussed.
\end{abstract}

Keywords: Complex TMOs, DFT, DMFT, Superstructures, Strongly Correlated System.

\section{INTRODUCTION}

The superstructure of perovskite materials have gained a lot of interest due to their promising applications for the electronic and transport properties as well as from its fundamental aspects. The transition metal oxides (TMOs) in the perovskites $\left(\mathrm{ABO}_{3}\right)$ geometry are smart materials found abundantly on the earth crusts. These materials are used for numerous applications in the field of electrical, electronics, medical, defense technology and etc. $[1,2]$. The modern technology assisted human civilizations through scientific revolution are being upgraded by exploring the smart materials. One of the categories of promising smart materials for the future is regarded as the TMOs in their nano-structures and superstructures [3]. The present study focused on the structural, electronic and transport properties of energetically stable superstructures $\mathrm{Ca}_{0.33} \mathrm{Sr}_{0.67} \mathrm{VO}_{3}$ and $\mathrm{Ca}_{0.67} \mathrm{Sr}_{0.33} \mathrm{VO}_{3}$.

The pristine $\mathrm{SrVO}_{3}$ is found in cubic phase, belongs to the space-group $\mathrm{Pm}-3 \mathrm{~m}$ with the Ca-atom sitting at the body center $(1 / 2,1 / 2,1 / 2)$ a. The transition metal, $\mathrm{V}$-atom is sitting at the origin $(0.0,0.0$, $0.0) \mathrm{a}$ and the three $\mathrm{O}$-atoms siting at the three face centers with Wyckoff's coordinates $(1 / 2,1 / 2,0.0) \mathrm{a}$, $(0.0,1 / 2,1 / 2) \mathrm{a}$, and $(1 / 2,0.0,1 / 2) \mathrm{a}[4]$.

Similarly, the pristine $\mathrm{CaVO}_{3}$ system in cubic phase belonging to the space-group Pm-3m with $\mathrm{V}$-atom at the body center $(1 / 2,1 / 2,1 / 2)$ a. The Ca-atom is sitting at the origin $(0.0,0.0,0.0)$ a and the three $\mathrm{O}$ atoms sitting with Wyckoff's coordinates at $(0.0$, $1 / 2,0.0) \mathrm{a},(0.0,0.0,1 / 2) \mathrm{a}$, and $(1 / 2,0.0,0.0) \mathrm{a}[4]$. The stable crystal structures for the pristine $\mathrm{CaVO}_{3}$ and the superstructure, $\mathrm{Ca}_{0.33} \mathrm{Sr}_{0.67} \mathrm{VO}_{3}$ are shown in fig.1 (a), (b).

The study of metal-to-insulator transition (MIT) caused by a strong electron correlation along with anomalous electronic properties in the metallic phase near the Mott transition, provide the various information regarding application in Mottronics, such as the volume resistive switching action, Mott FET, quantum gates etc. [5]. 
Though, MIT of the compounds can be studied through the hole or electron doping to the system either by band control or filling control methods [6]. Here, we used band controlling method to explain the MIT of experimentally synthesized compounds, $\mathrm{Ca}_{0.33} \mathrm{Sr}_{0.67} \mathrm{VO}_{3}$ by substituting Ca-ion in place of Sr-ion within the framework of $1 \times 1 \times 2$ supercell of $\mathrm{CaVO}_{3}$ as shown in Fig. 1 .

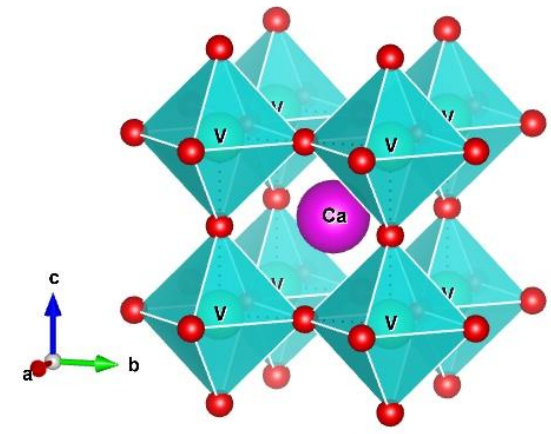

(a)

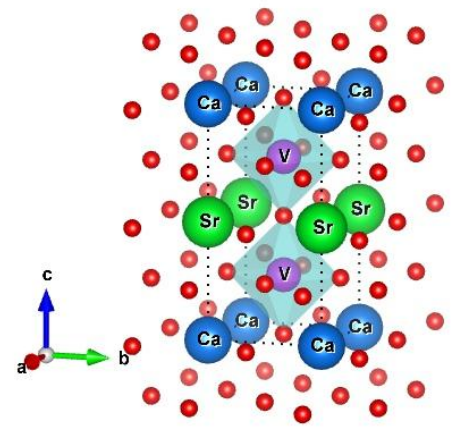

(b)

Fig.1: (a) (color online) The crystal structure of the pristine $\mathrm{CaVO}_{3}$ at which $\mathrm{V}$-ion is octahedrally co-ordinated by oxygen ligands (left) (b) the superstructure, $\mathrm{Ca}_{0.33} \mathrm{Sr}_{0.67} \mathrm{VO}_{3}$ (right)

The Mott transition behaviours of such systems are also studied using the density functional theory (DFT) and the dynamical mean field theory (DMFT) by various groups [7, 8]. A simple transition metal oxide system $\mathrm{Ca}_{1-\mathrm{x}} \mathrm{Sr}_{\mathrm{x}} \mathrm{VO}_{3}$ with a $3 \mathrm{~d}^{1}$ electronic configuration and a cubic perovskite lattice structure with the orthorhombically distorted $\mathrm{SrVO}_{3}$ upon increasing the Ca-doping have been investigated using LDA+DMFT [9-11].

In the present study, the stable structures, electronic properties are studied through conventional DFT and the MIT phase transition of pristine TMOs and their superstructures are studied through DMFT. The transport properties are also investigated through BoltzTrap frameworks (explained elsewhere). The materials under study are highly applicable for electrical, electronic, memory, and energy devices. The superstructure and nanostructure are of great interest of their Mottness, high efficiency of power conversion and high ZT factors. The Mott transition phenomena is useful for designing a leaky integrate and fire (LIF) artificial neurons [12-17], for neurocomputing, artificial neural network, machine learning etc.

Though, the MIT has various applications in different purposes, there is lack of systematic theoretical study compare to the experimental study. So, we are motivated to go more insight into the newly reconstructed superstructures for exploring MIT and thermoelectric properties.

Furthermore, the transition metal oxides have narrow conduction bands due to weak orbital overlap, which leads to localized electrons with low carrier mobilities. Transition metal oxides have recently been considered as thermoelectric (TE) materials that can operate at high temperature and they have their transport properties with high Seebeck coefficients (S) and low thermal conductivity $(K)$ and hence the higher value of figure of merit (ZT).

We have computed the thermal conductivity $K$, electrical conductivity $(\sigma / \tau)$, Seebeck coefficients $(\mathrm{S})$, and Figure of merit (ZT) for the designed superstructure [18] using semi-classical linearized Boltzmann transport equation.

\section{THEORETICAL, COMPUTATIONAL DETAILS AND EXPERIMENTAL INFORMATION}

\subsection{Theoretical Details:}

The electronic structure and transport properties of correlated electronic system are investigated employing ab-initio approaches through the full potential linearized augmented plane wave (FPLAPW) method with local orbitals (lo) [19, 20]. The optimized cubic phases of $\mathrm{CaVO}_{3}$ and $\mathrm{SrVO}_{3}$ and their superstructure framework are taken through the self-consistent calculation based on Kohn-Sham equation as given by,

$$
\left[-\frac{\hbar^{2}}{2 \mathrm{~m}} \nabla^{2}+\mathrm{v}_{\text {eff }}(\mathrm{r})\right] \psi_{\mathrm{i}}=\varepsilon_{\mathrm{i}} \psi_{\mathrm{i}}---(1)
$$


Where the effective Kohn-Sham potential is expressed as,

$$
\mathrm{v}_{\mathrm{eff}}(\mathrm{r})=\mathrm{v}(\mathrm{r})+\int e^{2} \frac{\left[\mathrm{n}_{\mathrm{e}}\left(\mathrm{r}^{\prime}\right)\right]}{\left|\mathrm{r}-\mathrm{r}^{\prime}\right|} \mathrm{dr}^{\prime}+\mathrm{v}_{\mathrm{xc}}(\mathrm{r})
$$

With, $v_{x c}(r)=\frac{\delta E\left[n_{e}(r)\right]}{\delta n_{e}(r)}$ is the exchange correlation potential with the probability density, $\mathrm{n}_{\mathrm{e}}(\mathrm{r})=\sum_{\mathrm{i}=1}^{\mathrm{N}}\left|\Psi_{\mathrm{i}}(\mathrm{r})\right|^{2}$.

The optimized value of the lattice parameter, $a$ is calculated using the concept of Birch-Murnaghan equation of state as given by,

$$
\mathrm{E}_{\text {tot }}(\mathrm{a})=\mathrm{E}_{0}+\frac{9 \mathrm{~V}_{0} \mathrm{~B}_{0}}{16}\left\{\left[\left(\frac{\mathrm{a}_{0}}{\mathrm{a}}\right)^{2}-1\right]^{3} \mathrm{~B}_{0}^{\prime}+\left[\left(\frac{\mathrm{a}_{0}}{\mathrm{a}}\right)^{2}-1\right]^{2}\left[6-4\left(\frac{\mathrm{a}_{0}}{\mathrm{a}}\right)^{2}\right]\right\}---(3)
$$

Where, $\mathrm{B}_{0}$ is the bulk modulus of elasticity at equilibrium (pressure $=0$ ), $\mathrm{V}_{0}$ is the equilibrium volume per atom/unit cell, $\mathrm{B}_{0}^{\prime}=\left(\frac{\partial \mathrm{B}}{\partial \mathrm{P}}\right)_{\mathrm{P}=0}$ and $\mathrm{E}_{0}$ is the total energy at equilibrium are treated as fitting parameters [20,21]. All the calculations were performed using the optimized parameters.
The theoretical prediction of transport coefficients of the TMOs are investigated for the optimized systems by using BoltzTraP code, a patching module of WIEN2k framework, which implements the linearized Boltzmann Transport Equation (BTE) $[18,22]$ as given by,

$$
\frac{\partial \mathrm{f}_{\mu}(\mathrm{T}, \mu)}{\partial \mathrm{t}}=-\mathrm{v}_{\alpha}(\mathrm{i}, \mathrm{k}) \cdot \frac{\partial \mathrm{f}_{\mu}(\mathrm{T}, \mu)}{\partial \mathrm{r}}-\frac{\mathrm{e}}{\hbar}\left(\mathrm{E}-\frac{1}{\mathrm{c}} \mathrm{v}_{\alpha}(\mathrm{i}, \mathrm{k}) \times \mathrm{H}\right) \cdot \frac{\partial \mathrm{f}_{\mu}(\mathrm{T}, \mu)}{\partial \mathrm{k}_{\alpha}}+\left.\frac{\partial \mathrm{f}_{\mu}(\mathrm{T}, \mu)}{\partial \mathrm{t}}\right|_{\text {scattering }}{ }^{---} \text {(4) }
$$

with $\mathrm{f}_{\mu}(\mathrm{T}, \mu)=\frac{1}{\exp ^{\left[\mu-E_{\mathrm{F}}\right]} / \mathrm{k}_{\mathrm{B}} \mathrm{T}^{+1}}$ is the Fermi-Dirac distribution for electron and $\mathrm{v}_{\alpha}(\mathrm{i}, \mathrm{k})=\frac{1}{\hbar} \frac{\partial \varepsilon_{\mathrm{i}, \mathrm{k}}}{\partial \mathrm{k}_{\alpha}}$ is the group velocity of the carriers.

For the figure of merit (ZT), the following equation is employed,

$$
\mathrm{ZT}=\frac{\sigma_{\alpha \beta} \mathrm{S}_{\alpha \beta}^{2}}{\kappa_{\alpha \beta}} \mathrm{T}---(5)
$$

Where, $\sigma_{\alpha \beta}(T, \mu)$ is the electrical conductivity tensor, $\kappa_{\alpha \beta}(\mathrm{T}, \mu)$ is the thermal conductivity tensor, $\mathrm{S}_{\alpha \beta}(\mathrm{T}, \mu)$ is the thermoelectric Seebeck coefficients, Where $\alpha$ and $\beta$ are tensor indices, $\mu$ and $N$ are the chemical potential and number of K-points implemented respectively [22].

Furthermore, the actual metal-insulator transition can be understood by using the dynamical mean field theory (DMFT), which starts from the Hubbard Hamiltonian [23-25] for single site interaction of opposite spin electrons as,

$$
\mathrm{H}=\sum_{\langle\alpha \beta\rangle} \mathrm{t}_{\alpha \beta} \mathrm{c}_{\alpha}^{\dagger} \mathrm{c}_{\beta}+\mathrm{U} \sum_{\alpha} \mathrm{n}_{\alpha \uparrow} \mathrm{n}_{\alpha \downarrow}---(6)
$$

Where, $t_{\alpha \beta}$ is tight-binding hopping amplitude between the lattice sites $\alpha$ and $\beta, c_{\alpha}^{\dagger}\left(c_{\alpha}\right)$ are the creation (annihilation) operators of electrons on localized orbitals on site, $\alpha$ and $n_{\alpha}=\mathrm{c}_{\alpha}^{\dagger} \mathrm{c}_{\alpha}$ is the localized occupation number on site, $\alpha$.

The DMFT maps the lattice problem (Hubbard Hamiltonian) of Eq. (6) onto a self-consistent auxiliary impurity problem, which is here solved numerically by the quantum Monte Carlo (QMC) technique, combined with the maximum entropy method [26], this technique allows us to calculate spectral functions.

The DMFT self-consistency cycle starts with an initial guess for the hybridization function, $\Delta\left(\mathrm{i} \omega_{\mathrm{n}}\right)=\sum_{v} \frac{\left|\mathrm{V}_{\mathrm{v}}\right|^{2}}{\mathrm{i} \omega_{\mathrm{n}}-\epsilon_{\mathrm{v}}}$

or bath Green's function,

$$
\mathcal{G}_{0}\left(\mathrm{i} \omega_{\mathrm{n}}\right)=\left[\mathrm{i} \omega_{\mathrm{n}}+\mu \Delta\left(\mathrm{i} \omega_{\mathrm{n}}\right)\right]^{-1}
$$

which determines the initial "bath" for the modified quantum impurity model.

Where, $V_{v}$ is the hybridization amplitude, $\epsilon_{v}$ is the bath energy level and $\left(i \omega_{n}\right)$ is the Matsubara frequency associated with the imaginary time, $(\tau)$. Using one of the quantum impurity solvers with the imaginary-time Green's function,

$$
\mathrm{G}(\tau)=-\left\langle\mathrm{T}_{\tau} \mathrm{c}(\tau) \mathrm{c}^{\dagger}(0)\right\rangle---(8)
$$


or its Fourier transform $\mathrm{G}\left(\mathrm{i} \omega_{\mathrm{n}}\right)$ and the Matsubara self-energy is,

$$
\Sigma\left(\mathrm{i} \omega_{\mathrm{n}}\right)=\mathcal{G}_{0}^{-1}\left(\mathrm{i} \omega_{\mathrm{n}}\right)-\mathrm{G}^{-1}\left(\mathrm{i} \omega_{\mathrm{n}}\right)---(9)
$$

are computed. This is actually the Fourier transform of Dyson equation, which computes the new bath Green's function, for the next iterative selfconsistent cycle [27-29]. The Green's function is then used for calculating the spectral function $\mathrm{A}(\mathrm{k}, \omega)$, which is the imaginary part of the singleparticle Green's function and therefore contains full information about the temporal and spatial evolution of a single electron/hole in the interacting many-electron system.

$$
\mathrm{A}(\mathrm{k}, \omega)=-\frac{1}{\pi} \operatorname{ImG}(\mathrm{k}, \omega)--(10)
$$

The spectral function $A(\omega)$ is analogous to the density of states(DOS) of conventional DFT.

\subsection{Computational Details}

The first-principles based approach has been used to deal with the structural, electronic properties of TMOs and their superstructures, employed on FPLAPW [30, 31]. The transport properties of the superstructures are calculated by solving the Boltzmann transport equation using BoltzTrap codes.

The conventional DFT is used for bandstructure and DOS calculation, which is used for the DMFT calculations. For the DFT calculation we have employed generalized gradient approximation (GGA) with the correlation functional as proposed by Perdew, Burke and Ernzerhof (PBE) exchange energy scheme [21,32].

The optimized parameters for the pristine systems and superstructures used for the present study are listed in the table 1 .

Table 1: The required values of lattice parameters, K-points, rKmax, Gmax and RMT* of the compounds with their space group used for the calculation.

\begin{tabular}{|c|l|l|l|l|l|l|c|}
\hline S.N. & \multicolumn{1}{|c|}{$\begin{array}{c}\text { Name of } \\
\text { system } \\
\text { group }\end{array}$} & $\begin{array}{c}\text { Space } \\
\text { Optimized } \\
\text { constant }\end{array}$ & K-points & rKmax & Gmax & RMT \\
\hline 1. & $\mathrm{CaVO}_{3}$ & $\begin{array}{l}p m-3 m \\
(221)\end{array}$ & $3.65 \AA$ & 800 & $3.97 \AA$ & 14 & $\begin{array}{l}\mathrm{Ca}=2.42, \mathrm{~V}= \\
1.79 \text { and } \mathrm{O}=1.62\end{array}$ \\
\hline 2. & $\mathrm{SrVO}_{3}$ & $\begin{array}{l}p m-3 m \\
(221)\end{array}$ & $3.86 \AA$ & 750 & $3.97 \AA$ & 16 & $\begin{array}{l}\mathrm{Sr}=2.46, \mathrm{~V}= \\
1.83 \text { and } \mathrm{O}=1.65\end{array}$ \\
\hline 3. & $\mathrm{Ca}_{0.33} \mathrm{Sr}_{0.67} \mathrm{VO}_{3}$ & $\begin{array}{l}P 4 / m m m \\
(123)\end{array}$ & $\begin{array}{l}\mathrm{a}=\mathrm{b}= \\
3.96{ }^{0} \mathrm{~A} \text { and } \\
\mathrm{c}=7.93 \AA\end{array}$ & 800 & $3.97 \AA$ & 16 & $\begin{array}{l}\mathrm{Ca}=2.50, \mathrm{Sr}= \\
2.50=\mathrm{V}=1.95 \\
\text { and } \mathrm{O}=1.77\end{array}$ \\
\hline 4. & $\mathrm{Ca}_{0.67} \mathrm{Sr}_{0.33} \mathrm{VO}_{3}$ & $\begin{array}{l}P 4 / \mathrm{mmm} \\
(123)\end{array}$ & $\begin{array}{l}\mathrm{a}=\mathrm{b}= \\
3.95^{\circ} \mathrm{A} \text { and c } \\
=7.91 \AA\end{array}$ & 750 & $3.95 \AA$ & 15 & $\begin{array}{l}\mathrm{Ca}=2.50, \mathrm{Sr}= \\
2.50, \mathrm{~V}=1.95 \\
\text { and } \mathrm{O}=1.77\end{array}$ \\
\hline
\end{tabular}

$* \mathrm{RMT}=$ Muffin-Tin radius of the elements

(All the values are consistent with the experimental results)

The Monk-horst pack of $11 \times 11 \times 5 \mathrm{k}$-mesh grid is used for the superstructures. The DMFT calculations are performed for various sets of coulombian interaction, $U$ and the thermodynamical parameter, $\beta$ in order to identify the critical value of MIT for each of the superstructures [33-35]. The energy and charge convergence criteria for the entire system are $10^{-5}$ $\mathrm{eV}$ and $10^{-3} \mathrm{e}$ with force convergence $0.05 \mathrm{eV} /{ }^{0} \mathrm{~A}$.

\subsection{Experimental Information}

The single crystals of superstructures, $\mathrm{Ca}_{1-\mathrm{x}} \mathrm{Sr}_{\mathrm{x}} \mathrm{VO}_{3}(\mathrm{x}=0,0.25,0.5,1)$ have grown for studying optical conductivity using a triple spectrometer, Photometrics and the optical reflectivity measurements using a Michelsontype Fourier-transform infrared spectrometer [36]. The spectra of $\mathrm{SrVO}_{3}$ and $\mathrm{CaVO}_{3}$ are also studied with bulk-sensitive high-resolution PES and XAS [37]. The Mott-Hubbard insulating gap for different values of $U$ for vanadates and titanates system were also studied from the various groups [38-40].

These experimental facts motivate us to perform the theoretical study to go more insight into the electronic and transport properties used for various applications in Mottronics. 


\section{RESULTS AND DISCUSSION}

\subsection{Structural Stability}

The structural stability of the compounds $\mathrm{CaVO}_{3}$, $\mathrm{SrVO}_{3}$ are calculated through energy minimization process. The value of the optimized lattice parameter for pristine $\mathrm{CaVO}_{3}$ and $\mathrm{SrVO}_{3}$ are found to be consistent with the experimental results [41, 42]. The reconstructed superstructure, $\mathrm{Ca}_{\mathrm{x}} \mathrm{Sr}_{(1-\mathrm{x})} \mathrm{VO}_{3}$ $(\mathrm{x}=0.33,0.67)$ using the base of $1 \times 1 \times 2$ supercell of $\mathrm{SrVO}_{3}$ and the site substitution of $\mathrm{Sr}$-atoms by $\mathrm{Ca}$-atoms on the supercell have been studied for their various properties.

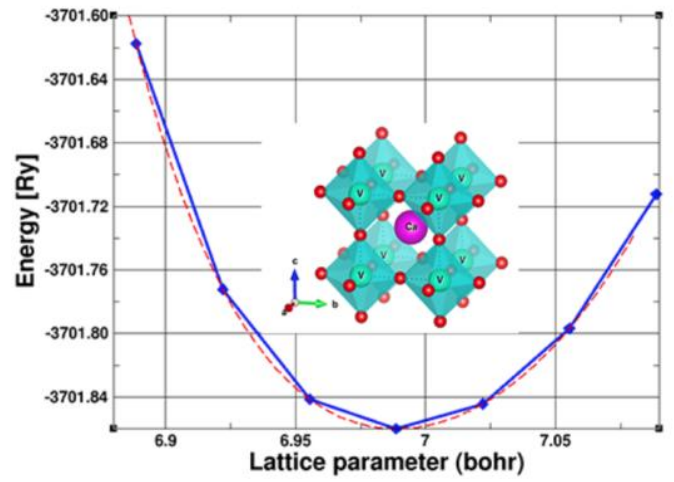

(a)
These superstructures with space-group $\mathrm{P} 4 / \mathrm{mmm}$, are also examined their stability through energy minimization process. The optimized lattice parameters of the superstructures, $\mathrm{Ca}_{0.33} \mathrm{Sr}_{0.67} \mathrm{VO}_{3}$ and $\mathrm{Ca}_{0.67} \mathrm{Sr}_{0.33} \mathrm{VO}_{3}$ are obtained and tabulated as in table-1.

The optimized structure of the sample $\mathrm{CaVO}_{3}$ and $\mathrm{Ca}_{0.67} \mathrm{Sr}_{0.33} \mathrm{VO}_{3}$ are shown in inset along with the energy vs. lattice parameter plot as shown in Fig. 2.

The lattice parameters of the superstructures are yet to be verified by experimentation.

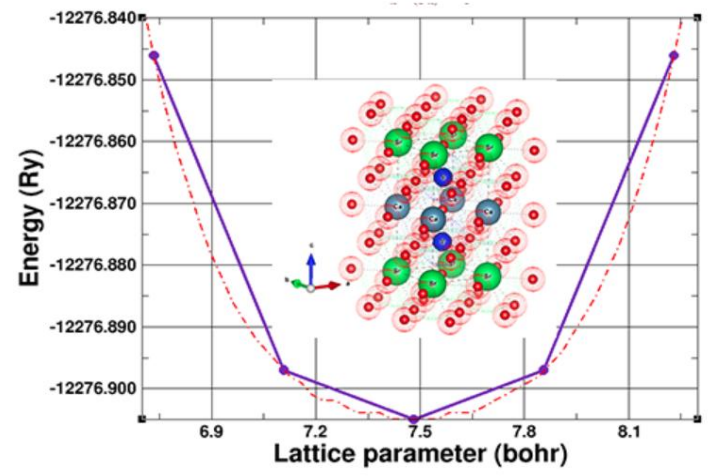

(b)

Fig. 2: (color online) The crystal structures (inset) and lattice parameter optimization curves for

(a) $\mathrm{CaVO}_{3}$ system (b) $\mathrm{Ca}_{0.33} \mathrm{Sr}_{0.67} \mathrm{VO}_{3}$ system.

\subsection{Electronic Structure of $\mathrm{Ca}_{\mathrm{x}} \mathrm{Sr}_{(1-\mathrm{x})} \mathrm{VO}_{3}$ System} 3.2.1 The Band structure and DOS

The bandstructure and the DOS plots of $\mathrm{CaVO}_{3}$ and $\mathrm{SrVO}_{3}$ systems are found to be well agreed with the previously calculated theoretical and experimental results. Both of these systems are

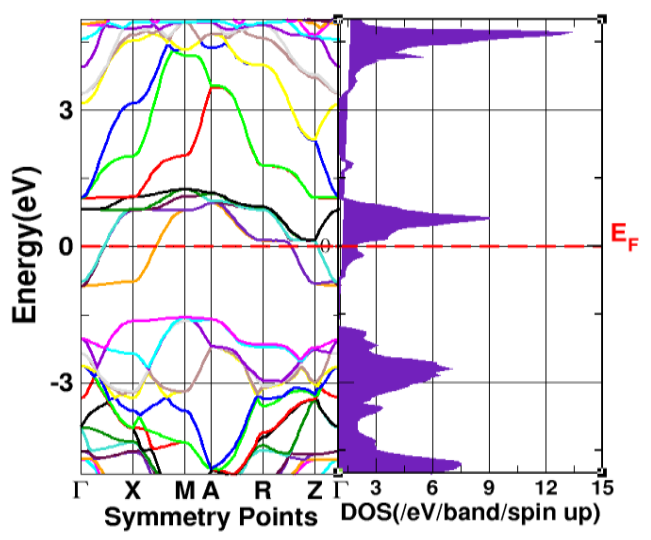

(a) correlated metals [45].

The combined plot of bandstructure and DOS of $\mathrm{Ca}_{0.33} \mathrm{Sr}_{0.67} \mathrm{VO}_{3}$ and $\mathrm{Ca}_{0.67} \mathrm{Sr}_{0.33} \mathrm{VO}_{3}$ are shown in Fig. 3 (a) and (b) indicating that these superstructures are also found to be correlated metal from the conventional DFT calculation.

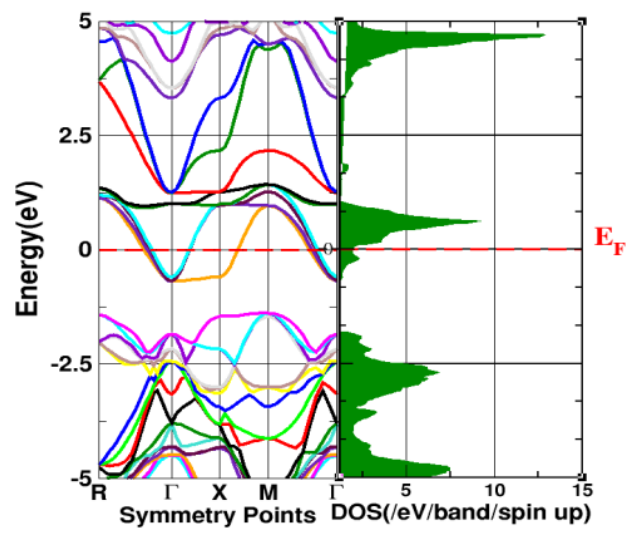

(b)

Fig. 3: (color online) The comparison of bandstructure and DOS for spin up channels of (a) $\mathrm{Ca}_{0.33} \mathrm{Sr}_{0.67} \mathrm{VO}_{3}$ system, and (b) $\mathrm{Ca}_{0.67} \mathrm{Sr}_{0.33} \mathrm{VO}_{3}$ system. 
Furthermore, the asymmetric distribution of DOS in spin up and spin down channels (Fig. 4) for the both superstructures $\mathrm{Ca}_{0.33} \mathrm{Sr}_{0.67} \mathrm{VO}_{3}$ and $\mathrm{Ca}_{0.67} \mathrm{Sr}_{0.33} \mathrm{VO}_{3}$ indicating that they show magnetism in ferromagnetic orderings. The magnetic moments are found to be $1.47 \mu_{\mathrm{B}}$ and $0.78 \mu_{\mathrm{B}}$ for $\mathrm{Ca}_{0.33} \mathrm{Sr}_{0.67} \mathrm{VO}_{3}$ and $\mathrm{Ca}_{0.67} \mathrm{Sr}_{0.33} \mathrm{VO}_{3}$ systems respectively.
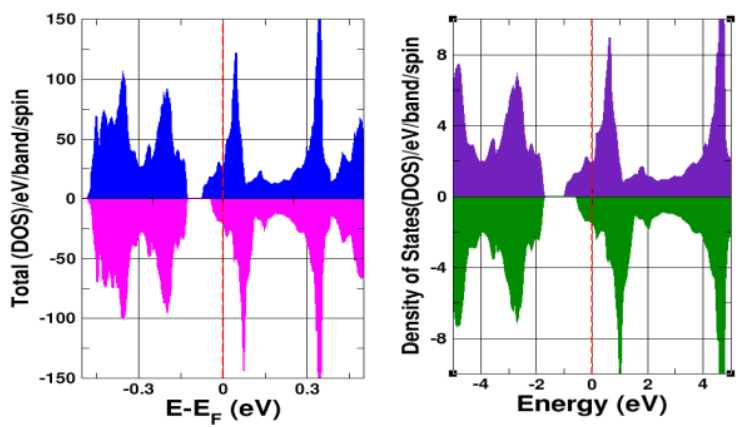

(b) (a)

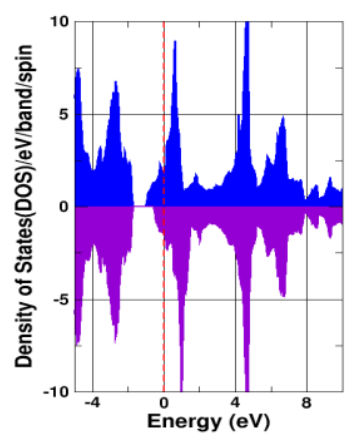

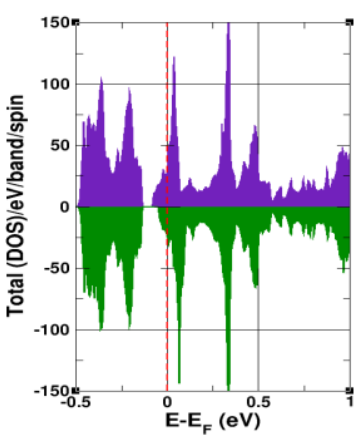

Fig. 4: (color online) The DOS plots for

(a) $\mathrm{Ca}_{0.33} \mathrm{Sr}_{0.67} \mathrm{VO}_{3}$ and (b) $\mathrm{Ca}_{0.67} \mathrm{Sr}_{0.33} \mathrm{VO}_{3}$ systems.

\subsubsection{The Charge Density Map and Fermi} Surface of the $\mathrm{Ca}_{\mathrm{x}} \mathrm{Sr}_{(1-\mathrm{x})} \mathrm{VO}_{3}$ :

The charge density contour plot shows that the $\mathrm{Ca}_{0.33} \mathrm{Sr}_{0.67} \mathrm{VO}_{3}$ system have a strong covalent bonding between $\mathrm{V}$ and $\mathrm{O}$-atoms which is mainly due to $\mathrm{p}-\mathrm{d}$ hybridization of $\mathrm{O}-2 \mathrm{p}$ and $\mathrm{V}-3 \mathrm{~d}$ orbitals as shown in Fig. 5(a) [46-49].

The Fermi surface plot (in inset) with band crossing by Fermi level of superstructures also supports the electronic structure calculations as shown in Fig. 5 (b) $[50,51]$.

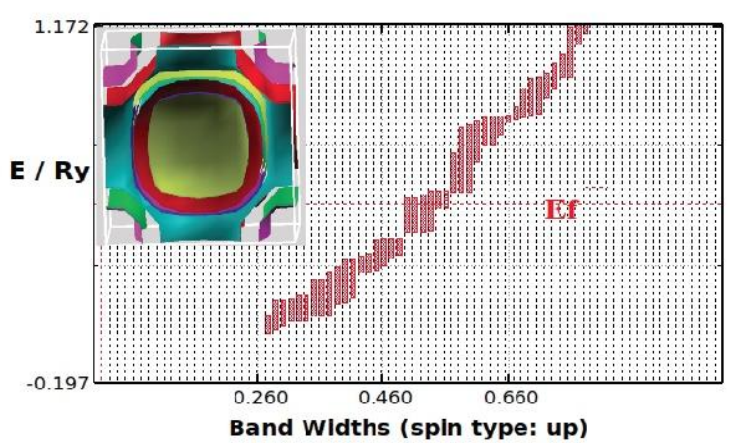

(b)

Fig. 5: (color online) (a) The 2D-contour plot of charge density distributions showing the bonding between neighboring atoms (b) The Fermi-surface (in inset) showing band crossing of Fermi level with 30-65 band levels.

\subsection{The Dynamical Mean Field Theory (DMFT) calculation:}

The Mott-Hubbard metal-insulator transition phenomena of the strongly correlated system are investigated using the numerical simulation of the data obtained from the conventional DFT calculation.

In general, the conventional DFT (GGA) underestimates the electronic bandstructure of strongly correlated system. Thus, we have used the dynamical mean field theory (DMFT) along with the DFT for the realistic calculation of electronic structure of superstructures [52 - 54]. From the DMFT calculations, the value of $U$ and $\beta$ required for Mott-Hubbard band splitting are calculated with the help the Green's function vs. imaginary time and its Fourier transform plots as shown in Fig. 6(a), (b). 


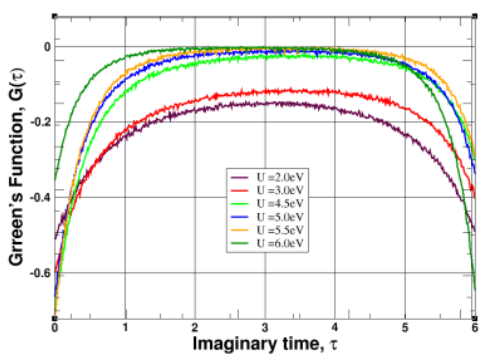

(a)

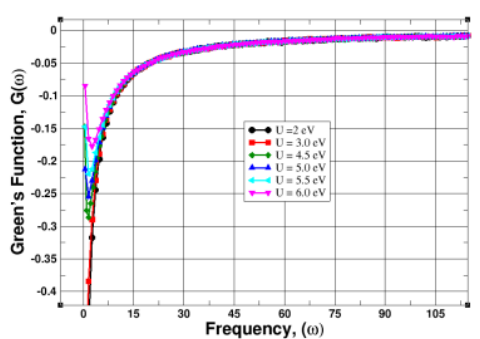

(b)

Fig. 6: (color online) (a) The variation of Green's function $\mathrm{G}_{\uparrow}(\tau)$ with imaginary time (b) The plot of Fourier transform of Green's function, $\mathrm{G}_{\uparrow}(\omega)$ [54].

The characteristic variation of Green function of imaginary time, $\mathrm{G}_{\uparrow}(\tau)$ with the imaginary time $(\tau)$ for various values of $U$, for the superstructure of $\mathrm{Ca}_{\mathrm{x}} \mathrm{Sr}_{(1-\mathrm{x})} \mathrm{VO}_{3}$ system is shown in Fig. 6(a) and the corresponding variation of spectral density, $\mathrm{A}(\omega)$ with the frequency $(\omega)$ is shown in Fig. 6(b), which is obtained by using the maximum entropy method of data analysis algorithm[ 55,56].

Moreover, Fig. 6 shows that the Mott-Hubbard band splitting starts from $\mathrm{U}=4.5 \mathrm{eV}$ for the value of $\beta=6(\mathrm{eV})^{-1}$. The corresponding spectral density plot of $\mathrm{G}_{\uparrow}(\tau)$ for the superstructure, $\mathrm{Ca}_{0.33} \mathrm{Sr}_{0.67} \mathrm{VO}_{3}$ with $\beta=6(\mathrm{eV})^{-1}$ and various values of $U$ is shown in Fig.7 (a).

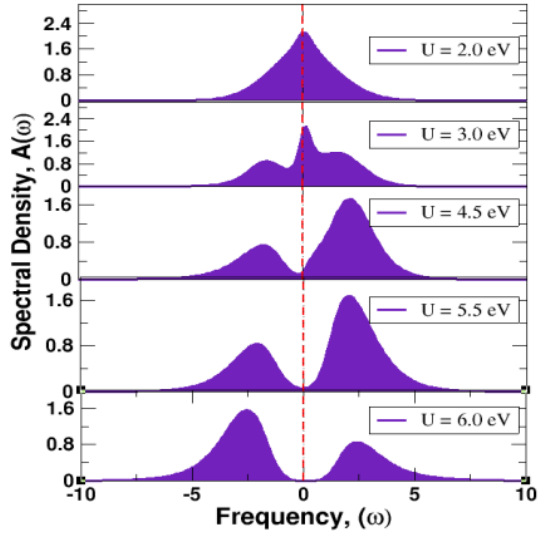

(a)

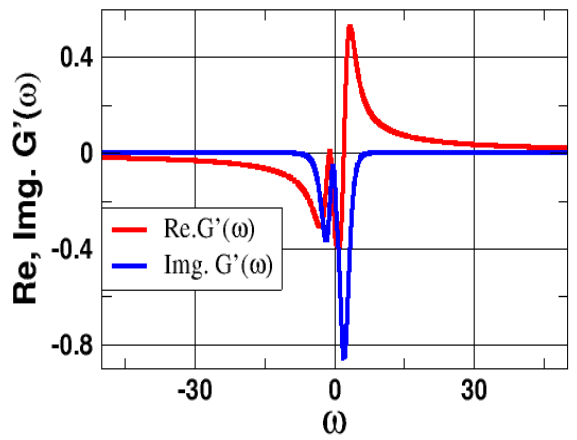

(b)

Fig. 7: (color online) (a) The spectral density vs. frequency for showing metal-insulator transition (MIT) with the variation of $U$ for $\beta=6(e V)^{-1}$. (b) The plot of real and imaginary part of retarded Green's function vs. frequency with $\mathrm{U}=4.5 \mathrm{eV}$ and $\beta=6(\mathrm{eV})^{-1}$.

The Mott-Hubbard splitting of $\mathrm{Ca}_{0.67} \mathrm{Sr}_{0.33} \mathrm{VO}_{3}$ system is obtained at $\mathrm{U}=4.5 \mathrm{eV}$ with $\beta=7(\mathrm{eV})^{-}$ ${ }^{1}$ (not shown in the graph).

Similarly, the corresponding real and imaginary part of retarded Green's function of frequency is shown in Fig. 7(b). In the Fermi liquid regime, the real part of the self energy leads to the shift of the non-interacting excitations, whereas the imaginary part is responsible for the broadening of the quasiparticle excitations. As the self-energy strongly depends on the frequency and in the case of Mott insulator, it will lead to a notable transfer of spectral weights.

The validity of spectral function, $\mathrm{A}(\omega)$ obtained from the maximum entropy model is checked through cross validation error (logistic regression analysis) as shown in fig. 8(a), (b) [56, 57].

From the sigmoid curve of logistic regression, the optimized value of $\alpha$ lies in the information-fitting region indicating that the data obtained by our calculation is realistic and information are close to the experimental results [58]. 


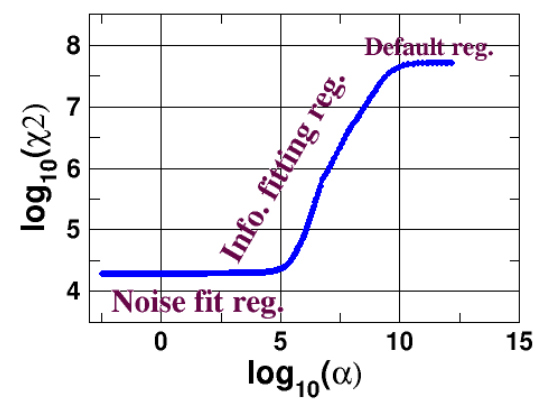

(a)

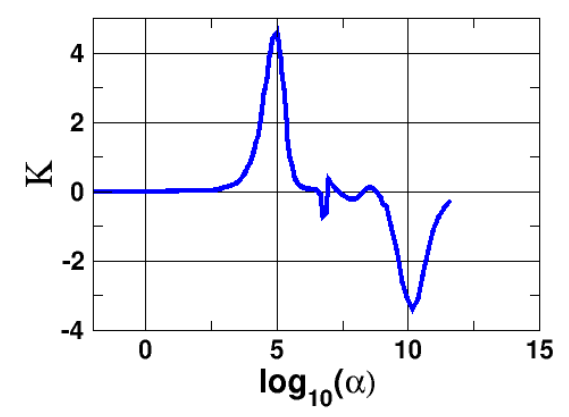

(b)

Fig. 8: (color online) (a) The logistic regression curve (sigmoid curve) for the optimized value of $\alpha$ (b) The error of logistic regression curve obtained by the curvature of the $\log _{10} \chi^{2}(K)$ versus $\log _{10}(\alpha)$.

3.4 Transport properties of for $\mathrm{Ca}_{\mathrm{x}} \mathrm{Sr}_{(1-\mathrm{x})} \mathrm{VO}_{3}$ system:

The transport properties of the superstructure are calculated using semiclassical linearized form of BTE through BoltzTrap frameworks implemented on ab-initio codes [59]. The graph showing the comparison of (a) Electrical conductivity $(\sigma / \tau)$ and (b) Thermal conductivity $(\kappa)$ at room temperature

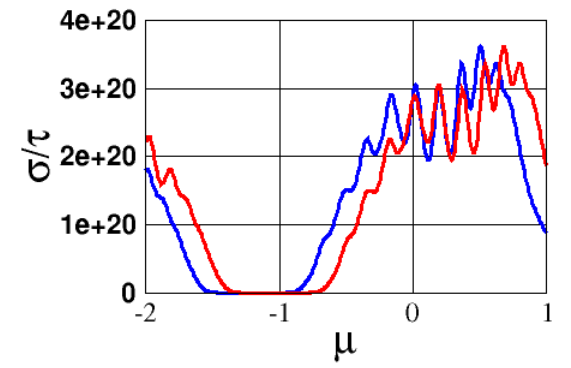

(a) are shown in fig. 9 (a), (b) for the superstructures, $\mathrm{Ca}_{0.33} \mathrm{Sr}_{0.67} \mathrm{VO}_{3}$ and $\mathrm{Ca}_{0.67} \mathrm{Sr}_{0.33} \mathrm{VO}_{3}$ with blue and red colors respectively. And the variation of Seebeck coefficient (S) with respect to the chemical potential, $(\mu)$ at room temperature for $\mathrm{Ca}_{0.33} \mathrm{Sr}_{0.67} \mathrm{VO}_{3}$ and $\mathrm{Ca}_{0.67} \mathrm{Sr}_{0.33} \mathrm{VO}_{3}$ systems are shown in Fig. 10 (a) with blue and red colors respectively $[18,58,59]$.

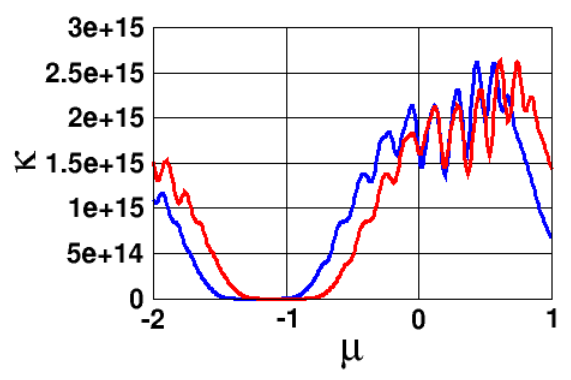

(b)

Fig. 9: (color online) (a) The variation of electrical conductivity $(\sigma / \tau)$ vs. $(\mu)(b)$ The variation of thermal conductivity $(\kappa)$ vs. $(\mu)$ for $\mathrm{Ca}_{0.33} \mathrm{Sr}_{0.67} \mathrm{VO}_{3}$ and $\mathrm{Ca}_{0.67} \mathrm{Sr}_{0.33} \mathrm{VO}_{3}$ with blue and red colors respectively $[59,60]$.

From the study of thermoelectric properties the figure of merit (ZT) factor for the superstructure $\mathrm{Ca}_{0.33} \mathrm{Sr}_{0.67} \mathrm{VO}_{3}$ with blue color and

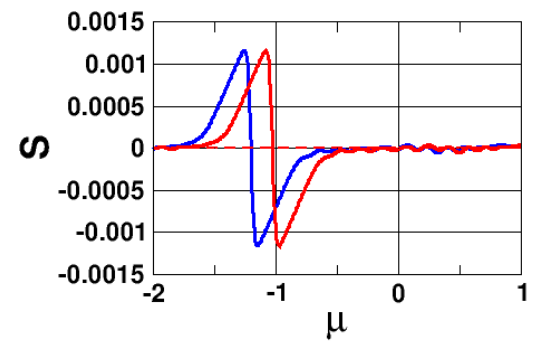

(a)
$\mathrm{Ca}_{0.67} \mathrm{Sr}_{0.33} \mathrm{VO}_{3}$ with red color are nearly equal to unity $(\approx 1)$ at near from $\mu=-1.266 \mathrm{eV}$ to $\mu=-0.938$ eV Fig. 10(b).

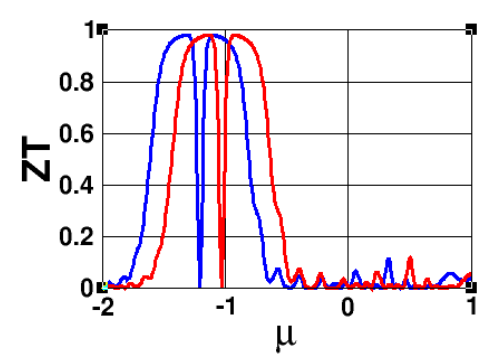

(b)

Fig. 10: (color online) (a) Variation of Seebeck coefficient $(S)$ with chemical potential $(\mu)$ (b) Figure of merit (ZT) at room temperature with chemical potential $(\mu)$ for superstructures, $\mathrm{Ca}_{0.33} \mathrm{Sr}_{0.67} \mathrm{VO}_{3}$ and $\mathrm{Ca}_{0.67} \mathrm{Sr}_{0.33} \mathrm{VO}_{3}$ with blue and red curves respectively [60-63]. 
The similar kind of thermoelectric properties are observed for both the superstructures, which can be used for the Mottronics applications.

\section{CONCLUSIONS}

The stability of the pristine and their superstructure are examined through energy minimization method. From the calculations, the lattice parameters for the stable superstructures, $\mathrm{Ca}_{0.33} \mathrm{Sr}_{0.67} \mathrm{VO}_{3}$ is found to be, $a=b=3.96 \quad \mathrm{~A}^{0}$ and $\mathrm{c}=7.93 \quad \mathrm{~A}^{0}$ and for $\mathrm{Ca}_{0.67} \mathrm{Sr}_{0.33} \mathrm{VO}_{3}$ is found to be, $\mathrm{a}=\mathrm{b}=3.95{ }^{\circ} \mathrm{A}$ and $\mathrm{c}$ $=7.91 \mathrm{~A}^{0}$. The Mott-Hubbard metal-insulator transition for their superstructure, $\mathrm{Ca}_{0.33} \mathrm{Sr}_{0.67} \mathrm{VO}_{3}$ and $\mathrm{Ca}_{0.67} \mathrm{Sr}_{0.33} \mathrm{VO}_{3}$ are obtained at $\mathrm{U}=4.5 \mathrm{eV}$ with $\beta=6(\mathrm{eV})^{-1}$ and $\mathrm{U}=4.5 \mathrm{eV}$ with $\beta=7(\mathrm{eV})^{-1}$ respectively, through DFT and DMFT with the continuous time quantum Monte Carlo (CT-QMC)hybridization method. This clearly indicates that both compounds are useful for Mottronics application. Furthermore, the ZT factor of the superstructures are approximately unity $(\sim 1)$. The estimated values of Seebeck coefficients and transport coefficients for the superstructure framework reveal that they can be used as the promising candidates for thermoelectric materials.

\section{ACKNOWLEDGEMENT}

The authors are gratefully acknowledged to UGC and NAST for financial supports. We thankfully acknowledge to supercomputer center (HPC) of KU for supporting in part through computational facility. NPA of the central department of Physics (CDP), T.U. is acknowledged for computational resources.

Editor's Note: This manuscript was submitted to Association of Nepali Physicists in America (ANPA) Conference 2020 for publication in special issue of Journal of Nepal Physical Society.

\section{REFERENCES}

[1] Imada, M.; Fujimori, A.; \& Tokura, Y. Metalinsulator transitions. Reviews of modern physics, 70(4): 1039 (1998).

[2] Morosan, E.; Natelson, D.; Nevidomskyy, A. H.; \& Si, Q. Strongly correlated materials. Advanced Materials, 24(36): 4896-4923 (2012).

[3] Nekrasov, I. A.; Keller, G.; Kondakov, D. E.; Kozhevnikov, A. V.; Pruschke, T.; Held, K.; \& Anisimov, V. I. Comparative study of correlation effects in $\mathrm{CaVO}_{3}$ and $\mathrm{SrVO}_{3}$. Physical Review B, 72(15): 155106 (2005).
[4] Piskunov, S.; Heifets, E.; Eglitis, R. I.; \& Borstel, G. Bulk properties and electronic structure of $\mathrm{SrTiO}_{3}, \mathrm{BaTiO}_{3}, \mathrm{PbTiO}_{3}$ perovskites: an ab initio HF/DFTstudy. Computational Materials Science, 29(2): 165-178 (2004).

[5] Son, J.; Rajan, S.; Stemmer, S.; \& James Allen, S. A heterojunction modulation-doped Mott transistor. Journal of Applied Physics, 110(8): 084503 (2011).

[6] Makino, H.; Inoue, I. H.; Rozenberg, M. J.; Aiura, Y.; Hase, I.; \& Onari, S. Band-width control in a perovskite-type $3 \mathrm{~d}^{1}$ correlated metal $\mathrm{Ca}_{1-\mathrm{x}} \mathrm{Sr}_{\mathrm{x}} \mathrm{VO}_{3}$. II. Optical spectroscopy investigation. arXiv preprint cond-mat/9801086 (1998).

[7] Inoue, I. H.; Morikawa, K.; Fukuchi, H.; Tsujii, T.; Iga, F.; \& Nishihara, Y. Metal-Insulator Transition in Nearly Stoichiometric $\mathrm{CaVO}_{3}$. Japanese Journal of Applied Physics, 32(S3): 451 (1993).

[8] Rozenberg, M. J.; Inoue, I. H.; Makino, H.; Iga, F.; \& Nishihara, Y. Low Frequency Spectroscopy of the Correlated Metallic System $\mathrm{Ca}_{\mathrm{x}} \mathrm{Sr}_{1-}$ ${ }_{\mathrm{x}} \mathrm{VO}_{3}$. Physical review letters, 76(25): 4781 (1996).

[9] Maiti, K.; Sarma, D. D.; Rozenberg, M. J.; Inoue, I. H.; Makino, H.; Goto, O.; \& Cimino, R. Electronic structure of $\mathrm{Ca}_{1-}{ }_{\mathrm{x}} \mathrm{Sr}_{\mathrm{x}} \mathrm{VO}_{3}$ : A tale of two energy scales. EPL (Europhysics Letters), 55(2): 246 (2001).

[10] Pavarini, E.; Yamasaki, A.; Nuss, J.; \& Andersen, O. K. How chemistry controls electron localization in $3 \mathrm{~d}^{1}$ perovskites: a Wannierfunction study. New Journal of Physics, 7(1): 188 (2005).

[11] Sondhi, S. L.; Girvin, S. M.; Carini, J. P.; \& Shahar, D. Continuous quantum phase transitions. Reviews of modern physics, 69(1): 315 (1997).

[12] Si, Q.; Rabello, S.; Ingersent, K.; \& Smith, J. L. Locally critical quantum phase transitions in strongly correlated metals. Nature, 413(6858): 804-808 (2001).

[13] Mott, N. F. Reviews of Modern Physics, 40: 677683 (1968).

[14] Pickett, M. D.; Medeiros-Ribeiro, G.; \& Williams, R. S. A scalable neuristor built with Mott memristors. Nature materials, 12(2): 114117 (2013).

[15] Sangwan, V. K.; Jariwala, D.; Kim, I. S.; Chen, K. S.; Marks, T. J.; Lauhon, L. J.; \& Hersam, M. C. Gate-tunable memristive phenomena mediated by grain boundaries in single-layer $\mathrm{MoS}_{2}$. Nature nanotechnology, 10(5): 403-406 (2015).

[16] Kravtsov, K.; Fok, M. P.; Rosenbluth, D.; \& Prucnal, P. R. Ultrafast all-optical implementation of a leaky integrate-and-fire neuron. Optics 
express, 19(3): 2133-2147(2011).

[17] Kurenkov, A.; Dutta Gupta, S.; Zhang, C.; Fukami, S.; Horio, Y.; \& Ohno, H. Artificial neuron and synapse realized in an antiferromagnet/ferromagnet heterostructure using dynamics of spin-orbit torque switching. Advanced Materials, 31(23): 1900636 (2019).

[18] Saeed, Y. Tuning the Transport Properties of Layered Materials for Thermoelectric Applications using First-Principles Calculations (Doctoral dissertation) (2014).

[19] Kaphle, G. C.; Ganguly, S.; Banerjee, R.; Khanal, R.; \& Mookerjee, A. "A study of magnetism in disordered Pt-Mn, Pd-Mn and Ni-Mn alloys: an augmented space recursion approach. Journal of Physics Condensed Matter, 24(29): 295501, (2012).

[20] Sholl, D.; \& Steckel, J. A. Density functional theory: a practical introduction. John Wiley \& Sons (2011).

[21] Martin, R. M. Electronic structure: basic theory and practical methods. Cambridge university press (2004).

[22] Adewale, A. A., et al. "Thermoelectric Transparent Properties of $\mathrm{SrTiO}_{3}$ Doped with Pm." Solid State Phenomena, Trans Tech Publications, 280 (2018).

[23] Anderson, P. W. Localized magnetic states in metals. Physical Review, 124(1): 41 (1961).

[24] Hubbard, J. Electron Correlations in Narrow Energy Bands, Proc. Roy. Soc. A 276, 238 (1963).

[25] Lancaster, T.; \& Blundell, S. J. Quantum field theory for the gifted amateur. OUP Oxford (2014).

[26] Skilling, J. (Ed.). Maximum Entropy and Bayesian Methods: Cambridge, England, 1988. Springer Science \& Business Media, 36 (2013).

[27] White, S. R.; Scalapino, D. J.; Sugar, R. L.; \& Bickers, N. E. Monte Carlo calculation of dynamical properties of the two-dimensional Hubbard model. Physical review letters, 63(14): 1523(1989).

[28] Sondhi, S. L.; Girvin, S. M.; Carini, J. P.; \& Shahar, D. Continuous quantum phase transitions. Reviews of modern physics, 69(1): 315(1997).

[29] Pavarini, E.; Koch, E.; Vollhardt, D.; \& Lichtenstein, A. (Eds.). Dmft at 25: Infinite dimensions: Lecture notes of the autumn school on correlated electron. Forschungszentrum Jülich, 4 (2014).

[30] Schwarz, K.; and Peter B. "Solid state calculations using WIEN2k." Computational Materials Science, 28(2): 259 (2003).

[31] Kaphle, G. C.; Adhikari, N.; \& Mookerjee, A.
Study of Spin Glass Behavior in Disordered $\mathrm{Pt}_{\mathrm{x}}$ $\mathrm{Mn}_{1-\mathrm{x}}$ Alloys: An Augmented Space Recursion Approach. Advanced Science Letters, 21(9): (2015).

[32] Kohn, W.; \& Sham, L. J. Self-consistent equations including exchange and correlation effects. Physical review, 140 (4A): A1133 (1965).

[33] Held, K.; Andersen, O. K.; Feldbacher, M.; Yamasaki, A.; \& Yang, Y. F. Bandstructure meets many-body theory: the LDA+ DMFT method. Journal of Physics: Condensed Matter, 20(6): 064202 (2008).

[34] Inoue, I. H.; Hase, I.; Aiura, Y.; Fujimori, A.; Haruyama, Y.; Maruyama, T.; \& Nishihara, Y. Systematic Development of the Spectral Function in the $3 \mathrm{~d}^{1}$ Mott-Hubbard System $\mathrm{Ca}_{1-\mathrm{x}} \mathrm{Sr}_{\mathrm{x}} \mathrm{VO}_{3}$. Physical review letters, 74(13): 2539 (1995).

[35] Pavarini, E.; Yamasaki, A.; Nuss, J.; \& Andersen, O. K. How chemistry controls electron localization in $3 \mathrm{~d} 1$ perovskites: a Wannierfunction study. New Journal of Physics, 7(1): 188 (2005).

[36] Makino, H.; Inoue, I. H.; Rozenberg, M. J.; Iga, F.; Aiura, Y.; \& Onari, S. Optical spectra of the correlated metallic system $\mathrm{Ca}_{1-} \mathrm{Sr}_{\mathrm{x}} \mathrm{VO}_{3}$. Physica B: Condensed Matter, 237: 56-58 (1997).

[37] Makino, H.; Inoue, I. H.; Rozenberg, M. J.; Hase, I.; Aiura, Y.; \& Onari, S. Bandwidth control in a perovskite-type $3 \mathrm{~d}$ 1-correlated metal $\mathrm{Ca}_{1-} \mathrm{x}$ $\mathrm{Sr}_{\mathrm{x}} \mathrm{VO}_{3}$. II. Optical spectroscopy. Physical Review B, 58(8): 4384 (1998).

[38] Sekiyama, A.; Fujiwara, H.; Imada, S.; Suga, S.; Eisaki, H.; Uchida, S. I.; \& Keller, G. Mutual Experimental and Theoretical Validation of Bulk Photoemission Spectra of $\mathrm{Sr}_{1-} \mathrm{Ca}_{\mathrm{x}} \mathrm{VO}_{3}$. Physical review letters, 93(15): 156402 (2004).

[39] Liebsch, A. Surface versus Bulk Coulomb Correlations in Photoemission Spectra of $\mathrm{SrVO}_{3}$ and $\mathrm{CaVO}_{3}$. Physical review letters, 90(9): 096401 (2003).

[40] Inoue, I. H.; Bergemann, C.; Hase, I.; \& Julian, S. R. Fermi Surface of $3 \mathrm{~d}^{1}$ Perovskite $\mathrm{CaVO}_{3}$ near the Mott Transition. Physical review letters, 88(23): 236403 (2002).

[41] Bocquet, A. E.; Mizokawa, T.; Morikawa, K.; Fujimori, A.; Barman, S. R.; Maiti, K., \& Onoda, M. Electronic structure of early 3d-transitionmetal oxides by analysis of the $2 p$ core-level photoemission spectra. Physical Review B, 53(3): 1161 (1996).

[42] Eguchi, R.; Kiss, T.; Tsuda, S.; Shimojima, T.; Mizokami, T.; Yokoya, T.; \& Watanabe, S. Bulkand surface-sensitive high-resolution photoemission study of two Mott-Hubbard systems: $\mathrm{SrVO}_{3}$ and $\mathrm{CaVO}_{3}$. Physical review letters, 96(7): 076402 (2006).

[43] Mossanek, R. J. O.; Abbate, M.; Yoshida, T.; 
Fujimori, A.; Yoshida, Y.; Shirakawa, N.; \& Vicentin, F. C. Evolution of the spectral weight in the Mott-Hubbard series $\mathrm{SrVO}_{3}-\mathrm{CaVO}_{3}-\mathrm{LaVO}_{3}$ $\mathrm{YVO}_{3}$. Physical Review B, 78(7): 075103 (2008).

[44] Mete, E. Electronic properties of transition metal oxides (Doctoral dissertation, METU) (2003).

[45] Mizutani, U. Introduction to the electron theory of metals. Cambridge University Press (2001).

[46] Sekiyama, A.; Fujiwara, H.; Imada, S.; Suga, S.; Eisaki, H.; Uchida, S. I.; \& Keller, G. Mutual Experimental and Theoretical Validation of Bulk Photoemission Spectra of $\mathrm{Sr}_{1-} \mathrm{Ca}_{\mathrm{x}} \mathrm{VO}_{3}$. Physical review letters, 93(15): 156402 (2004).

[47] Hussain, M. I.; Khalil, R. A.; Hussain, F.; Imran, M.; Rana, A. M.; \& Kim, S. Investigations of structural, electronic and optical properties of TM-GaO3 $(\mathrm{TM}=\mathrm{Sc}, \mathrm{Ti}, \mathrm{Ag})$ perovskite oxides for optoelectronic applications: a first principles study. Materials Research Express, 7(1): 015906 (2020).

[48] Joshy, L. D.; Rai, R. K.; \& Kaphle, G. C. Study of Electronic Properties and Chemical Bonding of Perovskite $\mathrm{LaFeO}_{3}$. International Technology and Science Publication, 3(1): 28-35 (2019).

[49] Paudyal, D.; Pecharsky, V. K.; Gschneidner Jr., K. A.; \& Harmon, B. N. Electron correlation effects on the magnetostructural transition and magnetocaloric effect in $\mathrm{Gd}_{5} \mathrm{Si}_{2} \mathrm{Ge}_{2}$. Physical Review B, 73(14): 144406 (2006).

[50] Veit, M. J., et al. "Three-dimensional character of the Fermi surface in ultrathin $\mathrm{LaTiO}_{3} / \mathrm{SrTiO}_{3}$ heterostructures." Physical Review B 99(11): 115126 (2019).

[51] Kachhava, C. M. Solid state physics. McGrawHill Publishing Company Ltd. (1992).

[52] Held, K.; Nekrasov, I. A.; Keller, G.; Eyert, V.; Blümer, N.; McMahan, A. K.; \& Vollhardt, D. Realistic investigations of correlated electron systems with LDA+ DMFT. Physica status solidi (b), 243(11): 2599 (2006).

[53] Gull, E. Continuous-time quantum Monte Carlo algorithms for fermions (Doctoral dissertation, ETH Zurich) (2008).

[54] Haule, Kristjan. "Quantum Monte Carlo impurity solver for cluster dynamical mean-field theory and electronic structure calculations with adjustable cluster base." Physical Review B, 75(15):155113 (2007).

[55] Rubtsov, A. N.; Savkin, V. V.; \& Lichtenstein, A. I. Continuous-time quantum Monte Carlo method for fermions. Physical Review B, 72(3): 035122 (2005).

[56] Bergeron, D. and Tremblay, A. M. S. Phys. Rev. E 94, 023303 (2016).

[57] Van Campenhout, J.; \& Cover, T. Maximum entropy and conditional probability. IEEE
Transactions on Information Theory, 27(4): 483489 (1981).

[58] Levy, R.; LeBlanc, J. P. F.; \& Gull, E. Implementation of the maximum entropy for analytic continuation. Computer Physics Communications, 215: 149-155 (2017).

[59] Madsen, G. K.; Carrete, J.; \& Verstraete, M. J. BoltzTraP $_{2}$, a program for interpolating band structures and calculating semi-classical transport coefficients. Computer Physics Communications, 231: 140-145 (2018).

[60] Liu, W.; Zhang, Q.; Yin, K.; Chi, H.; Zhou, X.; Tang, X.; \& Uher, C. High figure of merit and thermoelectric properties of Bi-doped $\mathrm{Mg}_{2} \mathrm{Si}_{0.4} \mathrm{Sn}_{0.6}$ solid solutions. Journal of Solid State Chemistry, 203: 333-339 (2013).

[61] Ge, Z. H.; Zhao, L. D.; Wu, D.; Liu, X.; Zhang, B. P.; Li, J. F.; \& He, J. Low-cost, abundant binary sulfides as promising thermoelectric materials. Materials Today, 19(4): 227-239 (2016)

[62] LaLonde, A. D.; Pei, Y.; Wang, H.; \& Snyder, G. J. Lead telluride alloy thermoelectrics. Materials today, 14(11): 526-532 (2011).

[63] Sclauzero, G.; Dymkowski, K.; \& Ederer, C. Tuning the metal-insulator transition in $\mathrm{d} 1$ and $\mathrm{d}$ 2 perovskites by epitaxial strain: A firstprinciples-based study. Physical Review B, 94(24): 245109 (2016).

[64] Vaz, C. A. F.; Hoffman, J.; Segal, Y.; Reiner, J. W.; Grober, R. D.; Zhang, Z.; \& Walker, F. J. Origin of the magnetoelectric coupling effect in $\mathrm{Pb}\left(\mathrm{Zr}_{0.2} \mathrm{Ti}_{0.8}\right) \mathrm{O}_{3} / \mathrm{La}_{0.8} \mathrm{Sr}_{0.2} \mathrm{MnO}_{3} \quad$ multiferroic heterostructures. Physical review letters, 104(12): 127202(2010).

[65] Anisimov, V. I.; Kondakov, D. E.; Kozhevnikov, A. V.; Nekrasov, I. A.; Pchelkina, Z. V.; Allen, J. W.; \& Sekiyama, A. Full orbital calculation scheme for materials with strongly correlated electrons. Physical Review B, 71(12): 125119 (2005)

[66] Nekrasov, I. A.; Held, K.; Blümer, N.; Poteryaev, A. I.; Anisimov, V. I.; \& Vollhardt, D. Calculation of photoemission spectra of the doped Mott insulator using LDA+ DMFT (QMC). The European Physical Journal B-Condensed Matter and Complex Systems, 18(1): 55-61 (2000).

[67] Fujimori, A.; Hase, I.; Nakamura, M.; Namatame, H.; Fujishima, Y.; Tokura, Y.; \& Strebel, O. Doping-induced changes in the electronic structure of $\mathrm{La}_{\mathrm{x}} \mathrm{Sr}_{1-\mathrm{x}} \mathrm{TiO}_{3}$ : Limitation of the oneelectron rigid-band model and the Hubbard model. Physical Review B, 46(15): 9841 (1992).

[68] Solovyev, I.; Hamada, N.; \& Terakura, K. $t_{2 g}$ versus all 3d localization in $\mathrm{LaMO}_{3}$ perovskites $(\mathrm{M}=\mathrm{Ti}-\mathrm{Cu})$ : First-principles study. Physical Review B, 53(11): 7158 (1996). 
[69] Huai-Hong, G.; Teng, Y.; Peng, T.; \& Zhi-Dong, Z. Theoretical study of thermoelectric properties of $\mathrm{MoS}_{2}$. Chinese Physics B, 23(1): 017201 (2013).

[70] Yakoubi, A.; Baraka, O.; \& Bouhafs, B. Structural and electronic properties of the Laves phase based on rare earth type $\mathrm{BaM}_{2}(\mathrm{M}=\mathrm{Rh}$, Pd,Pt). Results in Physics, 2: 58-65 (2012).

[71] Antonov, V.; Harmon, B.; \& Yaresko, A. Electronic structure and magneto-optical properties of solids. Springer Science \& Business Med [69] Sachdev, S. Quantum criticality: competing ground states in low dimensions. Science, 288 (5465): 475-480 (2000).

[72] Jaiswal, A.; Roy, S.; Srinivasan, G.; \& Roy, K. Proposal for a leaky-integrate-fire spiking neuron based on magnetoelectric switching of ferromagnets. IEEE Transactions on Electron Devices, 64(4): 1818-1824 (2017).

[73] Zhong, Z.; Wallerberger, M.; Tomczak, J. M.; Taranto, C.; Parragh, N.; Toschi, A.; \& Held, K. Electronics with correlated oxides: $\mathrm{SrVO}_{3} / \mathrm{SrTiO}_{3}$ as a Mott transistor. Physical review letters, 114(24): 246401 (2015).

[74] Wang, L.; Li, Y.; Bera, A.; Ma, C.; Jin, F.; Yuan, K.; \& Prellier, W. Device performance of the mott insulator $\mathrm{LaVO}_{3}$ as a photovoltaic material. Physical Review Applied, 3(6): 064015 (2015).

[75] Zubko, Pavlo, et al. "Interface physics in complex oxide heterostructures." Annu. Rev. Condens. Matter Phys. 2(1): 141 (2011).

[76] Tokura, Y. Critical features of colossal magnetoresistive manganites. Rep. Prog. Phys., 69(3): 797 (2006).
[77] Scafetta, Mark Dominic. Optical Properties and Electronic Structure of non-d $d^{0}$ Perovskite Oxide Epitaxial Films and Heterostructures. Drexel University (2015).

[78] Poizot, P. L. S. G.; Laruelle, S.; Grugeon, S.; Dupont, L.; \& Tarascon, J. M. Nano-sized transition-metal oxides as negative-electrode materials for lithium-ion batteries. Nature, 407(6803): 496-499 (2000).

[79] Ghimire, M. P.; Kaphle, G. C.; \& Thapa, R. K. Electronic and magnetic properties of double perovskites $\mathrm{Nd}_{2} \mathrm{MgIrO}_{6}$. Journal of Nepal Physical Society, 3(1): 50-54 (2015).

[80] Pandey, S.; Kaphle, G. C.; \& Adhikari, N. P. Electronic structure and magnetic properties of bulk elements ( $\mathrm{Fe}$ and $\mathrm{Pd}$ ) and ordered binary alloys (FePd and $\left.\mathrm{Fe}_{3} \mathrm{Pd}\right)$ : TB-LMTO-ASA. BIBECHANA, 11: 60-69 (2014).

[81] Yadav, D. K.; Bhandari, S. R.; Belbase, B. P.; Kaphle, G. C.; Rai, D. P.; \& Ghimire, M. P. Effects of electron-correlation, spin-orbit coupling, and modified Becke-Johnson potential in double perovskites SrLaBB' O6 $(\mathrm{B}=\mathrm{Ni}, \mathrm{Fe}$; $\left.\mathrm{B}^{\prime}=\mathrm{Os}, \mathrm{Ru}\right)$. Computational Materials Science, 170: 109168 (2019).

[82] Nepal, S.; Dhakal, R.; \& Galanakis, I. Ab initio study of the half-metallic full-Heusler compounds $\mathrm{Co}_{2} \mathrm{ZAl}[\mathrm{Z}=\mathrm{Sc}, \mathrm{Ti}, \mathrm{V}, \mathrm{Cr}, \mathrm{Mn}, \mathrm{Fe}]$; the role of electronic correlations. Materials Today Communications, 25: 101498 (2020).

[83] Abu-Mostafa, Y. S.; \& Psaltis, D. Optical neural computers. Scientific American, 256(3): 88-95 (1987). 